\title{
Serological and biological characteristics of "Streptococcus milleri" isolates from systemic purulent infections
}

\author{
K. KITADA, K. NAGATA*, T. YAKUSHIJI, H. EIFUKU and M. INOUE† \\ Department of Preventive Dentistry, Kagoshima University Dental School, 8-35-1 Sakuragaoka, Kagoshima 890 and \\ "Department of Clinical Laboratory, Tamana Central Hospital, 1950 Naka, Tamana City, Kumamoto 865, Japan
}

\begin{abstract}
Summary. Ninety-one Streptococcus milleri strains isolated from various systemic purulent lesions of 68 patients were examined by physiological and serological tests. Most strains formed a smooth colony (66 strains), did not form spontaneous aggregation of cells in BHI broth culture (79), were non- $\beta$-haemolytic ( $\alpha$ - 35 or non-41), and belonged to biotype Ia (49) or Ib (34) and to API taxa $S$. milleri I (41) or II (38). Almost all of the $\beta$-haemolytic strains as well as two-fifths of the non- $\beta$-haemolytic belonged to API taxon I; strains of API taxa II and III were non- $\beta$-haemolytic and non-haemolytic, respectively. Two-fifths (38) of the isolates belonged to one of eight serotypes, $a-g$ and $k$, and more than half (47) to Lancefield groups $\mathrm{A}, \mathrm{C}, \mathrm{F}$ or $\mathrm{G}$, the most frequent being type $b(19)$ and group $\mathrm{F}$ (33). Fifteen strains carried simultaneously type $a /$ group $\mathrm{A}, b / \mathrm{C}, c / \mathrm{C}, e / \mathrm{G}, f / \mathrm{F}$ or $k / \mathrm{G}$ antigens. Nineteen were neither typable nor groupable. All the 38 serotypable isolates were non- $\beta$-haemolytic and not members of API taxon III, and were serologically and physiologically similar to oral $S$. milleri. The isolates from various infected sites - sputum, thorax, abdomen, urogenitalia, skin, eye and dental — exhibited distinct combinations of biological and serological properties. These results suggest that serotyping, haemolytic properties and API taxon, and their combinations, would be useful methods to trace oral $S$. milleri in systemic infections.
\end{abstract}

\section{Introduction}

The epithet "Streptococcus milleri" is not included in the Approved List of Bacterial Names, ${ }^{1}$ and in the past 15 years the status of the taxonomy and nomenclature of these organisms has been debated. ${ }^{2}$ Several researchers have reported that the $S$. milleri group consists of only one species, $S$. milleri ${ }^{3}$ or Streptococcus anginosus ${ }^{4}$, whereas recent studies by other investigators have differentiated three taxa, $S$. anginosus, $S$. constellatus, and $S$. intermedius by phenotypic $^{2,5}$ and genotypical ${ }^{6}$ tests. However, the term $S$. milleri has been widely used, particularly by clinical microbiologists, to describe a bewildering assortment of streptococci associated with serious pyogenic infections. "S. milleri" group ${ }^{7}$ is one of the human commensals found most frequently in the mouth, gastrointestinal and genitourinary tracts. ${ }^{8,9}$ Several investigators have examined the association of indigenous $S$. milleri with various systemic purulent lesions in man, ${ }^{10-12}$ and demonstrated that $S$. milleri isolates from remote infectious and indigenous sites are often very similar on the basis of their Lancefield groups, biotypes and some physiological and biochem-

Received 16 Jan. 1991 ; accepted 8 March 1991.

+ Correspondence should be sent to Dr M. Inoue. ical characteristics. ${ }^{13-15}$ However, the strict microbiological relations between them have not been fully elucidated.

Recently, we have obtained large numbers of $S$. milleri-like isolates from various systemic infections. ${ }^{16}$ The objective of this study was to characterise these systemic isolates by various serological and biological typing systems, including those previously proposed by us. ${ }^{17-19}$ The results are then discussed in terms of the similarities of these strains to human oral isolates previously isolated and characterised. ${ }^{17,18}$

\section{Materials and methods}

\section{Isolation and identification}

$S$. milleri-like isolates were collected through routine microbiological tests from 70 purulent foci at various body sites of 68 patients at Tamana Central Hospital, Kumamoto, Japan; details are listed in table I. They were categorised into seven groups - sputum, thorax, abdomen, skin, urogenital, eye and dental-based upon the sites of origin. Samples from the thorax included purulent pleural fluid (in five cases) and pus from a pulmonary abscess; "abdomen" included purulent abdominal fluid (5), bile (4), pus from 
Table I. Sources of clinical samples and numbers of $S$. milleri isolates

\begin{tabular}{|c|c|c|c|c|}
\hline \multirow{2}{*}{ Origin } & \multicolumn{2}{|c|}{ Number of samples* } & \multirow{2}{*}{$\begin{array}{l}\text { Mean age [range] of } \\
\text { subjects } \\
\text { (years) }\end{array}$} & \multirow{2}{*}{ Number of isolates } \\
\hline & Male & Female & & \\
\hline Sputum & 16 & 2 & $61[25-87]$ & 23 \\
\hline Thorax & $3(2) \dagger$ & $3(1)$ & $58[39-65]$ & $11(7)$ \\
\hline Abdomen & 6 & $7(2)$ & $54[17-84]$ & $15(2)$ \\
\hline Skin & 8 & $7(2)$ & $50[7-71]$ & $19(2)$ \\
\hline Urogenital tract & 3 & $13(6)$ & $66[20-95]$ & $18(6)$ \\
\hline Eye & 1 & 0 & 65 & 2 \\
\hline Dental & 0 & 1 & 52 & 3 \\
\hline Total number & $37(2)$ & $33(11)$ & $58[7-95]$ & $91(17)$ \\
\hline
\end{tabular}

* Two isolates each were isolated from 15 samples and three each from three samples.

$\dagger(\mathrm{)}$, Isolates in pure culture.

abdominal abscesses (3) and ileo-caecal pus (1); "skin" included pus from post-operative wounds (7), subcutaneous abscesses (5), perianal abscesses (2) and a decubitus ulcer; "urogenital" included purulent urine (12) and pus from genital abscesses (4); "eye" denotes a purulent discharge from the conjunctiva; "dental" denotes a root abscess.

Of the 70 cultures, 13 from thorax, abdomen, skin and urogenital sites were pure cultures of $S$. millerilike cocci (table I). In the others, $S$. milleri-like cocci were isolated in association with other bacteria, mostly obligate anaerobes. The clinical significance of most of these cultures was reported previously. ${ }^{16}$

When the 70 cultures, which had been stored in $10 \%$ skim milk at $-20^{\circ} \mathrm{C}$, were subcultured on Columbia Basal Agar (BBL) containing sheep blood $5 \%$ (blood agar) anaerobically at $37^{\circ} \mathrm{C}$ for $48 \mathrm{~h}, 15$ cultures produced two different types of colony and three yielded three colony forms for each (smooth, rough, and smooth-variant). This provides the total of 91 isolates (table I). All isolates were identified as $S$. milleri by the API-20 STREP system (API System S.A., Montalieu Vercieu, France). They were maintained in Brain Heart Infusion Broth (BHI; Difco) containing glycerol $45 \%$ at $-20^{\circ} \mathrm{C}$ until used.

\section{Physiological and serological characterisation}

These were performed as outlined previously for oral isolates. ${ }^{17-19}$ Briefly, biotyping of the isolates was based upon their abilities to ferment maltose, salicin and lactose as proposed previously. ${ }^{17,19}$ Identification by the API 20-STREP system was performed according to the manufacturer's instructions. Haemolysis and colonial morphology were defined on blood agar. ${ }^{17}$ The isolates were also allocated to the taxa $S$. anginosus ( $\beta$-haemolytic; lactose fermenting or not), $S$. intermedius (non- $\beta$-haemolytic; lactose fermenting) or $S$. constellatus (non- $\beta$-haemolytic; lactose non-fermenting) according to the American CDC criteria. $^{2}$ Spontaneous cellular aggregation was examined macroscopically after cells were grown in BHI broth anaerobically at $37^{\circ} \mathrm{C}$ for $48 \mathrm{~h}$.

For serological characterisation, cell-surface carbo- hydrate antigen preparations were allowed to react with antisera in agar $1.2 \%$ gel as described previously. ${ }^{18}$ The antigens were extracted from whole cells grown on glucose-containing media by the method of Rantz and Randall. ${ }^{20}$ Typing antisera, anti- $a-k$, were prepared with the respective serotype reference strains of oral $S$. milleri. ${ }^{18,19}$ Lancefield group-specific antisera, $A-G$, were purchased from Difco.

\section{Results}

\section{General features of isolates}

Most (66) of the 91 isolates formed smooth colonies on blood agar. Only a few (12) produced spontaneous aggregation of cells in BHI broth culture; of these, all but two formed rough colonies on blood agar. Most of the strains isolated in pure culture (17) did not aggregate and formed smooth colonies.

The isolates were mostly non- $\beta$-haemolytic -35 were $\alpha$-haemolytic and 41 were non-haemolytic (table II). Most belonged to API taxa I (41) or II (38) and to biotypes Ia (49) or Ib (34) (table II). Nearly half of the isolates fermented lactose (51) and generally consisted of API II/biotype Ia; the 40 non-fermenting strains were API I or III/biotype Ib. Most $\beta$-haemolytic strains and $40 \%$ of the non- $\beta$-haemolytic strains belonged to API I; API II and III strains were non- $\beta$ haemolytic and non-haemolytic, respectively. According to American CDC nomenclature ${ }^{2} 47$ strainsmostly API II (35) or III (10) and biotype Ia [45]were $S$. intermedius, 29 strains-mostly API I (26) and biotype $\mathrm{Ib}(26)$ - were $S$. constellatus, and 15 strainsmostly lactose-non-fermenting (11), API I (13) and biotype Ib (8) or Ia (4) - were $S$. anginosus (table II).

Most isolates carried serotype antigens $(a-g$ or $k$; 38) or Lancefield group antigens (A, C, F or G; 47) (table III). Members of serotype $b$ (19), group F (33) and the untypable (un-cross-reactive)/ungroupable strains (19) were most common. All the strains of serotypes $a, c, f$ and $k$ carried, simultaneously, group antigens A, C, F and G, respectively. A few strains of the $a$-cross-reactive/group F, serotype $b /$ group $\mathrm{C}$, type 
Table II. Distribution of $S$. milleri isolates according to haemolysis and biotype

\begin{tabular}{|c|c|c|c|c|c|c|c|c|c|c|c|c|c|c|c|c|}
\hline \multirow{3}{*}{ Origin } & \multicolumn{16}{|c|}{ Number of isolates designated } \\
\hline & \multicolumn{3}{|c|}{ Haemolytic } & \multicolumn{3}{|c|}{ API taxon } & \multicolumn{7}{|c|}{ Biotype* } & \multicolumn{3}{|c|}{ CDC taxon } \\
\hline & $\beta-$ & $\alpha-$ & non- & I & II & III & Ia & $\mathrm{Ib}$ & Ic & Id & IIa & $\mathrm{IIb}$ & IId & S.a. & S.c. & S.i. \\
\hline Sputum & 9 & 7 & 7 & 14 & 8 & 1 & 11 & 11 & 0 & 0 & 0 & 0 & 1 & 9 & 7 & 7 \\
\hline Thorax & 0 & $4(1) \dagger$ & $7(6)$ & $9(7)$ & 2 & 0 & 2 & $8(6)$ & 0 & 0 & 0 & 0 & I (1) & 0 & $9(7)$ & 2 \\
\hline Abdomen & 3 & $8(1)$ & $4(1)$ & 5 & $10(2)$ & 0 & $8(2)$ & 4 & 1 & 1 & 0 & 1 & 0 & 3 & 3 & $9(2)$ \\
\hline Skin & 2 & $9(1)$ & $8(1)$ & $10(1)$ & $8(1)$ & 1 & $10(2)$ & 7 & 0 & 2 & 0 & 0 & 0 & 2 & 7 & $10(2)$ \\
\hline Urogenital & 1 & $5(1)$ & $12(5)$ & 3 & $5(1)$ & $10(5)$ & $13(6)$ & 4 & 0 & 0 & 1 & 0 & 0 & 1 & 3 & $14(6)$ \\
\hline Eye & 0 & 2 & 0 & 0 & 2 & 0 & 2 & 0 & 0 & 0 & 0 & 0 & 0 & 0 & 0 & 2 \\
\hline Dental & 0 & 0 & 3 & 0 & 3 & 0 & 3 & 0 & 0 & 0 & 0 & 0 & 0 & 0 & 0 & 3 \\
\hline $\begin{array}{l}\text { Total number } \\
\text { of isolates }\end{array}$ & 15 & $35(4)$ & $41(13)$ & $41(8)$ & $38(4)$ & $12(5)$ & $49(10)$ & $34(6)$ & 1 & 3 & 1 & 1 & $2(1)$ & 15 & $29(7)$ & $47(10)$ \\
\hline $\begin{array}{l}\text { Number of } \\
\text { positive clinical } \\
\text { samples§ }\end{array}$ & 13 & $29(4)$ & $32(10)$ & $29(4)$ & $33(4)$ & $11(5)$ & $40(10)$ & $24(3)$ & 1 & 3 & 1 & 1 & $2(1)$ & 13 & $19(3)$ & $40(10)$ \\
\hline
\end{tabular}

S.a.; S. anginosus; S. c.; S. constellatus; S.i.: S. intermedius.

* No biotype IIc strain was isolated; biotypes Ia, Ic, and IIa were lactose fermenting.

$\dagger()$, Isolated in pure culture.

$\S$ See table 1 .

Table III. Distribution of $S$. milleri isolates according to serovar

\begin{tabular}{|c|c|c|c|c|c|c|c|c|c|c|c|c|c|c|c|c|c|}
\hline \multirow{2}{*}{ Origin } & \multicolumn{17}{|c|}{ Number of isolates belonging to serovars* } \\
\hline & $a / \mathrm{A}$ & $b$ & $b / \mathrm{C}$ & $c / \mathrm{C}$ & $d$ & $e$ & $e / \mathrm{G}$ & $f / \mathrm{F}$ & $g$ & $k / \mathrm{G}$ & $\mathrm{C}$ & $\mathrm{F}$ & G & $a-/ \mathrm{F}$ & $b-$ & $g-$ & $-/-$ \\
\hline Sputum & 0 & 3 & 0 & 0 & 0 & 0 & 0 & 0 & 1 & 0 & 0 & 11 & 1 & 1 & 0 & 1 & 5 \\
\hline Thorax & 0 & $9(7) \dagger$ & $\mathbf{0}$ & 1 & 0 & 0 & 0 & 0 & 0 & 0 & 0 & 1 & 0 & 0 & 0 & 0 & 0 \\
\hline Abdomen & 1 & 1 & 1 & 1 & 0 & $2(1)$ & 1 & 0 & 0 & 0 & 0 & 2 & 1 & 0 & 0 & 0 & $5(1)$ \\
\hline Skin & 0 & $3(1)$ & 0 & 2 & $1(1)$ & 0 & 0 & 1 & 0 & 0 & 0 & 6 & 0 & 0 & 1 & 0 & 5 \\
\hline Urogenital & 0 & 2 & 0 & 0 & 0 & 1 & 0 & $2(1)$ & 0 & 0 & 1 & $7(3)$ & 0 & 1 & 0 & 0 & $4(2)$ \\
\hline Eye & 0 & 0 & 0 & 0 & 0 & 0 & 0 & 0 & 0 & 2 & 0 & 0 & 0 & 0 & 0 & 0 & 0 \\
\hline Dental & 0 & 0 & 0 & 2 & 0 & 0 & 0 & 1 & 0 & 0 & 0 & 0 & 0 & 0 & 0 & 0 & 0 \\
\hline $\begin{array}{l}\text { Total number } \\
\text { of isolates }\end{array}$ & 1 & $18(8)$ & 1 & 6 & $1(1)$ & $3(1)$ & 1 & $4(1)$ & 1 & 2 & 1 & $27(3)$ & 2 & 2 & 1 & 1 & $19(3)$ \\
\hline $\begin{array}{l}\text { Number of } \\
\text { positive clinical } \\
\text { samples§ }\end{array}$ & 1 & $10(4)$ & 1 & 4 & $1(1)$ & $3(1)$ & 1 & $4(1)$ & 1 & 1 & 1 & $23(3)$ & 2 & 1 & 1 & 1 & $17(3)$ \\
\hline
\end{tabular}

* Strains of serotypes $h, i$ and $j$ and Lancefield groups B, D and E were not isolated; $a / \mathrm{A}$, serotype $a /$ group A; $b / \mathrm{C}$, serotype $b /$ group C; $c / \mathrm{C}$, serotype $c /$ group $\mathrm{C} ; e / \mathrm{G}$, serotype $e /$ group $\mathrm{G} ; f / \mathrm{F}$, serotype $f /$ group $\mathrm{F} ; k / \mathrm{G}$, serotype $k /$ group $\mathrm{G} ; a-/ \mathrm{F}, a$-cross-reactive/group $\mathrm{F} ; b$ and $g-, b$ - and $g$-cross-reactive respectively; $-/-$, unreactive with any antisera.

† See () , isolated in pure culture.

$\S$ See table I.

$e /$ group $\mathrm{G}$, and the untypable/ungroupable but $b$ - or $g$ cross-reactive were also found.

\section{Relationship between biological and serological properties}

As summarised in table IV, strains that were non$\beta$-haemolytic, fermented lactose and were of API taxon II or biotype Ia consisted of many different serotypes or serogroups. In contrast, the $\beta$-haemolytic strains were confined to a few serovars, mostly to group F, and all except a few strains of the API I or III, biotype Ib and the lactose-non-fermenting strains belonged to the serovars $b, \mathrm{~F}$ or $-/-$

Of the 33 group $\mathrm{F}(\mathrm{F}, f / \mathrm{F}, a-/ \mathrm{F})$ strains, one-third (mainly F) was $\beta$-haemolytic and generally API I/ biotype Ib/lactose negative (S. anginosus). The remaining strains were non- $\beta$-haemolytic and generally $\mathrm{II} / \mathrm{Ia} /$ $+(S$. intermedius) although several non-haemolytic strains were III $/ \mathrm{Ia} /+$. All except a few strains of the other serovars were non- $\beta$-haemolytic. Generally, serotype $b(b, b / \mathrm{C}, b$-) strains (20) were $\mathrm{I} / \mathrm{Ib} /-(S$. constellatus). Some $\alpha$-haemolytic strains of the $-/$ groups (19) were also $\mathrm{I} / \mathrm{Ib} /-(S$. constellatus) but other (non-haemolytic) strains were II or III/Ia/ $+(S$. intermedius). Strains of the remaining serovars were $\mathrm{II} / \mathrm{Ia} /+(S$. intermedius $)$. All the 38 serotypable strains were non- $\beta$-haemolytic and not members of API III (i.e., they were $S$. intermedius API II or $S$. constellatus). $S$. intermedius strains were more serologically variable 
Table IV. Distribution of $S$. milleri isolates in the CDS $S$. milleri taxa in relation to their serological and physiological variations

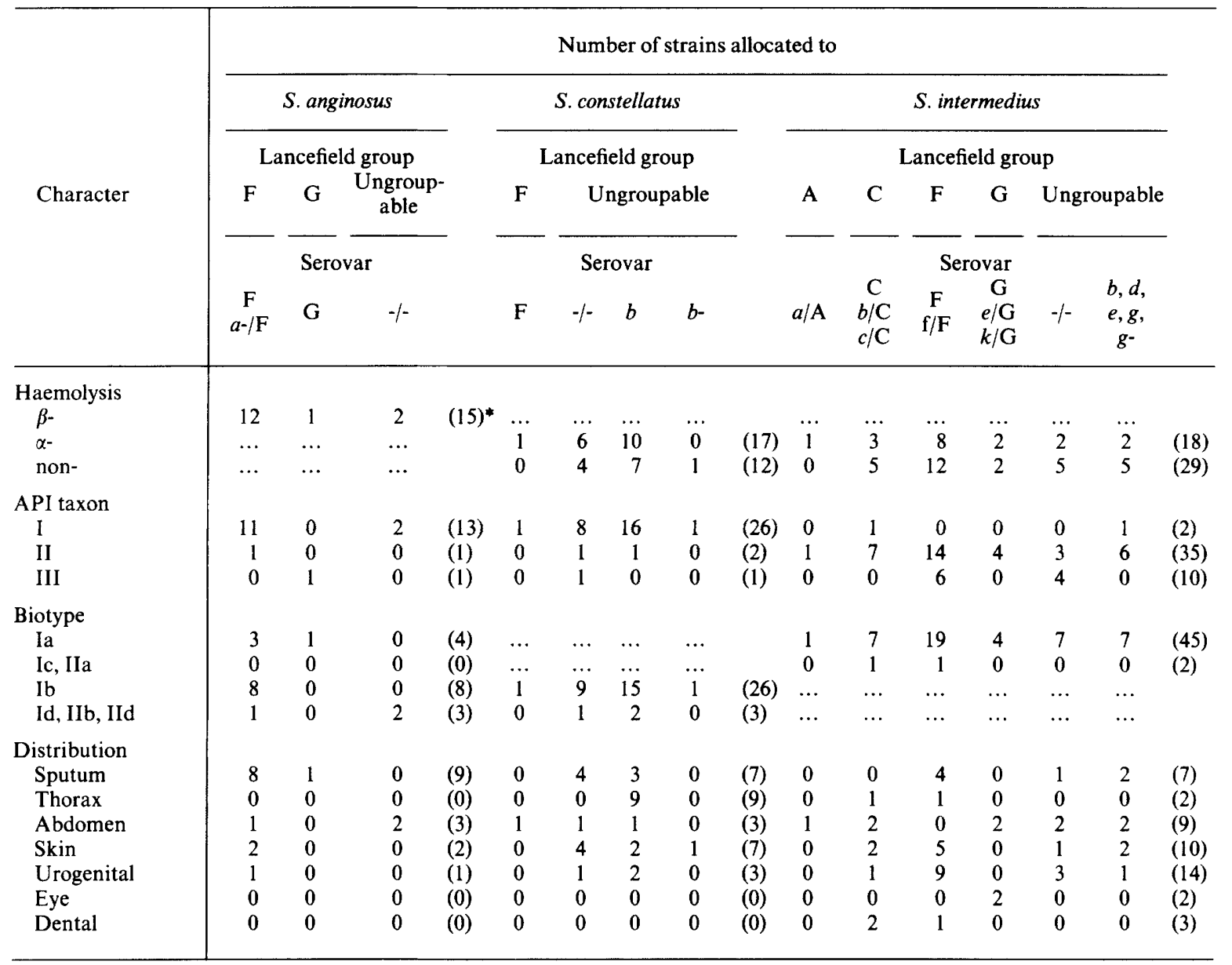

*(), Total number of strains.

than those of $S$. anginosus and $S$. constellatus (table IV).

\section{Variations in isolation sites}

Isolates from the five main anatomical sites (excluding the eye and dental isolates) varied significantly in their biological and serological properties. As summarised in tables II and IV, $-\beta$-haemolytic strains were predominant only in sputum, although non- $\beta$-haemolytic strains were also found at that site. The strains from the other sites were mainly non- $\beta$-haemolytic: $\alpha$ and non-haemolytic strains were isolated in equal proportions from thorax and skin; $\alpha$-haemolytic strains were isolated more commonly from abdominal sites and non-haemolytic strains from urogenital sites. The strains of API I (mostly biotype Ib/lactose negative) and II (mostly $\mathrm{Ia} /+$ ) were also detected in these sites; API III strains (mostly Ia/+) predominated only among the urogenital isolates. Thus, the bulk of the isolates from thorax, abdomen, and urogenital sites were non- $\beta$-haemolytic API I ( $S$. constellatus), $\alpha$ haemolytic II ( $S$. intermedius), and non-haemolytic III
( $S$. intermedius), respectively. The skin isolates comprised nearly equal proportions of non- $\beta$-haemolytic I ( $S$. constellatus) and II ( $S$. intermedius) and the sputum strains consisted of $\beta$-haemolytic I ( $S$. anginosus) as well as non- $\beta$-haemolytic I ( $S$. constellatus) and II ( $S$. intermedius).

As shown in tables III and IV, the thorax isolates were serologically most uniform (only three serovars) and were mainly serotype $b$ strains. Abdominal isolates showed the widest variation, consisting of nine serovars including the $-/-$ strains as a major group. The isolates from urogenital, skin and sputum sites were moderately variable, each of them consisting of seven serovars and predominated by group $\mathrm{F}$ and untypable (mostly -/-) strains. The few isolates from eye and dental sources were all $\mathrm{II} / \mathrm{Ia} /+(S$. intermedius $)$, but the former and the latter were $\alpha$ - and non-haemolytic, and serovars $k / \mathrm{G}$ and $c / \mathrm{C}$ or $f / \mathrm{F}$, respectively (tables II-IV).

All of the 17 strains isolated in pure culture were non- $\beta$-haemolytic, mostly non-haemolytic (table II). The pure isolates from each anatomical site were biologically and serologically simple (tables II and III); e.g., all of the thoracic pure strains were $\mathrm{I} / \mathrm{Ib} /$ - 
(S. constellatus) and serotype $b$ whereas the urogenital isolates were $\mathrm{III} / \mathrm{Ia} /+(S$. intermedius $)$ and group $\mathrm{F}$ or $-1$.

In two of the 68 patients examined, samples were obtained simultaneously from unrelated remote sites of the same individual. Such isolates were very similar biologically and serologically: the isolates from the sputum and the urine of one patient were $\beta$-haemolytic, of API I and biotype $\mathrm{Ib}$ ( $S$. anginosus), and of serovar $a-/ \mathrm{F}$; those from the sputum and the lung abscess of another patient were non-haemolytic and API I/ biotype Ib ( $S$. constellatus) although serological tests showed $b$ or $-/$.

\section{Discussion}

Despite much attention in recent years, the taxonomy and nomenclature of the $S$. milleri group have remained unresolved. In the American CDC system, ${ }^{2}$ this group of streptococci is differentiated into three taxa-S. anginosus, $S$. intermedius and $S$. constellatuson the basis of their haemolytic properties and lactose fermentation. Very recently, Whiley et al. ${ }^{5}$ have proposed a biochemical scheme for distinguishing among them, which is based on criteria different from the CDC scheme. At present, there is no widely accepted and established biochemical scheme for the $S$. milleri group. Therefore, we compared clinical isolates from various systemic infections principally on the basis of their individual biochemical and serological properties but not on their species or "species/taxon names".

Two-fifths of the $91 S$. milleri isolates from systemic pyogenic infections reacted with one of the 11 typing antisera raised against oral $S$. milleri strains (table III). The physiological characteristics of these serotypable pyogenic strains (table IV) were also very similar to those of the isolates from dental plaque of adults. ${ }^{17} \mathrm{In}$ particular, none of the serotypable strains isolated from systemic sites was $\beta$-haemolytic, nor did any belong to API taxon III or biotype Id. Both systemic and dental groups were very similar in the proportional distributions of the biotypes and the colonial morphologies and in the frequency and distribution of Lancefield groups. These results indicate that the isolates from systemic infections that react with the antisera against the oral $S$. milleri reference strains may originate in the oral cavity.

The $S$. milleri group has been demonstrated to be isolated frequently from thoracic infections in which the streptococcus is detectable in pure culture more frequently than in other infections (table I; Gossling ${ }^{8}$ ). It is notable that all except one of the thorax isolates, including all those isolated in pure culture, carried the type antigens detected in oral $S$. milleri strains, mostly $b$ antigen (table III); the type $b$ vaccine strain NCTC 10708 is an isolate from odontogenic abscess. ${ }^{18,21}$ These findings suggest that $S$. milleri isolates from thorax infections may originate in the oral cavity.
Many researchers have demonstrated that bacteraemia occurs frequently after various dental treatments, especially tooth extraction ${ }^{22-24}$ and that the bacterial composition in bacteraemia and dental plaques is generally very similar, ${ }^{25}$ indicating that the blood stream may be the vehicle for opportunist infections from the mouth to systemic sites. ${ }^{12,26}$

In contrast, the isolates from urogenital sites were mostly strains of API III (table II) and, generally, did not carry any type antigens found with oral isolates (table III). S. milleri strains isolated from the urogenital tract, vagina and urine have been reported to be physiologically distinct and significantly different from the isolates from other body sites and infectious foci, ${ }^{13,14,27}$ and it is proposed that they should be classified as $S$. milleri III. $^{28}$ Thus, the physiologically and serologically distinct urogenital isolates appear to originate in body sites other than the oral cavity or thorax. Isolates from sputum were generally untypable and frequently carried Lancefield group antigens (table III) but they often varied from urogenital isolates in API taxon and biotype (table II); they also varied from the thoracic/oral patterns described above, suggesting that such infections did not originate in the oral cavity.

There was very close correlation between haemolytic properties and API taxa among the $S$. milleri isolates from systemic infections (tables II and IV): most $\beta$-haemolytic strains belonged to API I, whereas API II and III strains were non- $\beta$-haemolytic and nonhaemolytic, respectively; the isolates from the individual sites of infection exhibited particular combinations of these biological properties. It has been shown that the main habitat of API III $S$. milleri (mostly nonhaemolytic; table II) is the urogenital tract and urine $^{13,28}$ whereas the oral inhabitants are mostly non- $\beta$-haemolytic API II $S$. milleri. ${ }^{17,19}$ Therefore, the combination of these two physiological characteristics could be used as a marker to determine the original habitats of strains among various body sites. Because oral $S$. milleri isolates exhibit marked serological similarity and specificity in individuals, ${ }^{19}$ serological characteristics of $S$. milleri isolates might provide useful information to determine their route of dissemination. In two individuals in the present study, isolates from remote body sites were similar in biological and serological properties.

Ruoff and $\mathrm{Kunz}^{28}$ have reported that, within the $S$. milleri group, API taxon I corresponds to $\beta$-haemolytic $S$. milleri ( $S$. anginosus), API II includes strains designated as either $S$. intermedius or $S$. constellatus, and API III corresponds to $S$. milleri strains referred to by Ball and Parker ${ }^{13}$ as "unidentified urine isolates". In the present study, the bulk of the urogenital $S$. milleri isolates were accordingly identified as API III ( $S$. intermedius); however, the majority of strains designated as $S$. constellatus and $S$. anginosus belonged to API I, and $S$. intermedius included strains of API III as well as API II. The reason for these differences remain unclear but might include small differences in 
the criteria used by researchers for differentiation within the $S$. milleri group.

We have demonstrated previously that strains of oral isolates of Lancefield groups A, C, F and G from young adults carry, simultaneously, serotype antigens $a, c, f$ and $k$, respectively. ${ }^{18}$ In the mouths of children, group $\mathrm{F}$ isolates that lack type $f$ antigens were also detected frequently. ${ }^{19}$ In the present study, new assortments of group and type antigen were found in some strains from systemic purulent infections, including $c, b / \mathrm{C}, \mathrm{G}, e / \mathrm{G}, a$ - and $b$ - (table III); we had not

\section{References}

1. Skerman VBD, McGowan V, Sneath PHA. Approved lists of bacterial names. Int J Syst Bacteriol 1980; 30: 225-420.

2. Facklam RR. The major differences in the American and British streptococcus taxonomy schemes with special reference to Streptococcus milleri. Eur J Clin Microbiol 1984; 3: 91-93.

3. Colman G, Williams REO. Taxonomy of some human viridans streptococci. In: Wannamaker LW, Matsen JM (eds) Streptococci and streptococcal diseases. New York Academic Press. 1972: 281-299.

4. Coykendall AL, Wesbecher PM, Gustafson KB. “Streptococcus milleri", Streptococcus constellatus, and Streptococcus intermedius are later synonyms of Streptococcus anginosus. Int J Syst Bacteriol 1987; 37: 222-228.

5. Whiley RA, Fraser H, Hardie JM, Beighton D. Phenotypic differentiation of Streptococcus intermedius, Streptococcus constellatus, and Streptococcus anginosus strains within the "Streptococcus milleri group". J Clin Microbiol 1990; 28 . 1497-1501.

6. Whiley RA, Hardie JM. DNA-DNA hybridization studies and phenotypic characteristics of strains within the 'Streptococcus milleri group'. J Gen Microbiol 1989; 135: 2623-2633.

7. Hardie JM. Oral streptococci. In: Sneath PHA, Mair NS, Sharpe ME, Holt JG (eds) Bergey's Manual of systematic bacteriology, vol 2. Baltimore, Williams and Wilkins. 1986: 1054-1063.

8. Gossling J. Occurrence and pathogenicity of the Streptococcus milleri group. Rev Infect Dis 1988; 10: 257-285.

9. Ruoff KL. Streptococcus anginosus ("Streptococcus milleri"): the unrecognized pathogen. Clin Microbiol Rev 1988; 1: 102108.

10. Parker MT, Ball LC. Streptococci and aerococci associated with systemic infection in man. J Med Microbiol 1976; 9 : 275-302.

11. Van der Auwera P. Clinical significance of Streptococcus milleri. Eur J Clin Microbiol 1985; 4: 386-390.

12. Admon D, Ephros MA, Gavish D, Raz R. Infection with Streptococcus milleri. J Infect 1987; 14: 55-60.

13. Ball LC, Parker MT. The cultural and biochemical characters of Streptococcus milleristrains isolated from human sources. J Hyg 1979; 82: 63-78.

14. Poole PM, Wilson G. Occurrence and cultural features of Streptococcus milleri in various body sites. J Clin Pathol 1979; 32: 764-768. encountered these with oral $S$. milleri isolates. Most commonly, however, the relationships between group and type antigens found in isolates from systemic purulent foci (table III) was similar to that seen in oral indigenous strains. ${ }^{18,19}$ As demonstrated in many other studies, ${ }^{8} S$. milleri bearing the group $\mathrm{F}$ antigen was the variety most frequently isolated whatever the clinical source.

This research was supported in part by Grant-in-Aid for Scientific Research no. 02771611 from the Ministry of Education, Science and Culture of Japan.

15. Unsworth PF. Hyaluronidase production in Streptococcusmilleri in relation to infection. J Clin Pathol 1989; 42: 506-510.

16. Nagata K. Clinical significance of Streptococcus milleri isolated from various suppurative lesions. Kansenshogaku Zasshi $1990 ; 64: 444-454$.

17. Yakushiji T, Katsuki M, Yoshimitsu A, Mizuno J, Inoue M. Isolation and physiological characterization of Streptococcus milleri strains from human dental plaque. Microbios 1988; 55: 161-171.

18. Yakushiji $\mathrm{T}$, Konagawa $\mathbf{R}$, Oda $\mathbf{M}$, Inoue $\mathbf{M}$. Serological variation of oral Streptococcus milleri. J Med Microbiol 1988; 27 : 145-151.

19. Yakushiji T, Kitada K, Okita Y, Inoue M. Distribution of Streptococcus milleri in the oral cavities of Japanese children. Microb Ecol Health Dis 1990; 3: 171-179.

20. Rantz LA, Randall E. Use of autoclaved extracts of hemolytic streptococci for serological grouping. Stanford Med Bull 1955; 13: 290-291.

21. Guthof O. Uber pathogene, "vergrünende Streptokokken" Streptokokken-Befunde bei dentogenen Abszessen und Infiltraten im Bereich der Mundhöhle. $\mathrm{Zbl}$ Bakteriol Parasitenkd Infektionskr Hyg Abt I Orig 1956; 166: 553564.

22. Phillips I, Warren C, Harrison JM, Sharples P, Ball LC, Parker MT. Antibiotic susceptibility of streptococci from the mouth and blood of patients treated with penicillin or lincomycin and clindamycin. J Med Microbiol 1976; 9 : 393-404.

23. Symington JM. Streptococci isolated from post-extraction bacteraemias. Br J Oral Surg 1975; 13: 91-94.

24. Guntheroth WG. How important are dental procedures as a cause of infective endocarditis? Am J Cardiol 1984; 54 797-801.

25. Crawford I, Russell C. Streptococci isolated from the bloodstream and gingival crevice of man. J Med Microbiol 1983 ; 16: $263-269$.

26. Murray HW, Gross KC, Masur H, Roberts RB. Serious infections caused by Streptococcus milleri. Am J Med 1978; 64: 759-764.

27. Ruoff KL, Kunz LJ. Identification of viridans streptococci isolated from clinical specimens. J Clin Microbiol 1982;15 920-925.

28. Ruoff KL, Kunz LJ. Use of the rapid STREP system for identification of viridans streptococcal species. $J$ Clin Microbiol 1983; 18: 1138-1140. 